\title{
Alkylglycerol/Rosemary Capsules
}

National Cancer Institute

\section{Source}

National Cancer Institute. Alkylglycerol/Rosemary Capsules. NCI Thesaurus. Code C156175.

An orally available capsule containing alkylglycerols and Rosmarinus officinalis extracts standardized to diterpenes, including carnosic acid and carnosol, with potential antineoplastic and anti-inflammatory activities. Upon administration, carnosic acid and carnosol may induce apoptosis by decreasing apoptosis regulator B-cell lymphoma 2 (Bcl-2) expression, decrease tumor cell growth through inhibition of mammalian target of rapamycin (mTOR) phosphorylation, and inhibit metastatic activity by preventing the adhesion of tumor cells to type I collagen and fibronectin. Additionally, these diterpenes may decrease inflammation by downregulating cyclooxygenase (COX) type 2 (COX-2) protein synthesis. Alkylglycerols (alkyl-Gro) are bioactive ether lipids that may, through a not yet fully elucidated mechanism, inhibit tumor cell growth and metastases. 